Mesdames, Messieurs, bonjour. J'appartiens donc au groupe St-Gobain, je ne suis pas du tout dans le secteur d'activités que vous partagez les uns et les autres; je vais peut être vous choquer avec mes propos, mais on m'a demandé de venir vous parler de choses peut être un petit peu différentes de ce que vous avez l'habitude d'entendre en matière d'approche.

Je vous parlerai d'analyse de la valeur, d'abord en ce qui concerne la méthodologie propre et puis, j'essaierai de vous donner quelques sujets de réflexions, quant à l'application de l'analyse de la valeur vis à vis des problèmes que pose l'eau, mais l'eau d’une façon générale.

Je suis dans le groupe St-Gobain depuis de nombreuses années, j'ai occupé plusieurs fonctions, d'abord au niveau du laboratoire de recherche et de développement, puis de construction d'usines et d'aménagement de sites et ce qui m'a amené a explorer différentes approches globales des problèmes et je considère que l'analyse de la valeur fait partie de ces méthodes globales permettant dans notre économie de marché de mieux connaître, de mieux apprécier les tenants et aboutissants d'un produit et je pense que plus nous irons vers des produits précis en fonction des besoins des utilisateurs, plus nous aurons besoin d'approcher, de façon pertinente, les fonctions que doivent remplir les produits.

Matière première noble dans tous les pays où elle est rare, voire absente, l'eau est devenue un produit banalisé, plus ou moins inconsciemment, comme un produit inépuisable donc, dont la valeur au sens large est très faible. Alors que nos hommes politiques s'inquiètent enfin des risques majeurs de pollution de ce bien indispensable à la vie, aussi bien animale que végétale, on ne pouvait trouver meilleur terrain d'application de l'analyse de la valeur pour valoriser et restituer à l'eau la place qui lui revient.

L'eau est indispensable à la vie.

○ Quel est le prix de la vie?

L'eau est partie prenante de bon nombre de technologies modernes.

○ Quel est le prix payé par l'utilisateur?

L'eau est abusivement utilisée pour éliminer bon nombre de déchets industriels.

- Quels sont les coûts de la dépollution ?

L'eau, élément essentiel de l'hygiène des hommes.

- Quel est le prix payé pour le

traitement des eaux usées?

Je pourrai continuer cette énumération avec ce style encore longtemps, mais je vais arrêter là pour dire simplement ceci. $\mathrm{Ne}$
Exposé de Monsieur R. NATTERO

Societé KERLANE

Président de l'Association française d'analyse de la valeur pour la Region P.A.C.A.

\section{ANALYSE DE LA VALEUR CREATIVITE ET COMMUNICATION}

doit-on pas considérer l'eau comme un produit énergétique ? A partir de ce moment là, ses rapports avec la société et les hommes seraient posés en terme de valeur globale, s'inscrivant dans le cycle de nature, vie moderne, à laquelle nous adhérons tous à des degrés divers, certes.

Enfin, pour gérer au mieux nos ressources en eau, il faut que les solutions retenues passent par des analyses objectives, des fonctions remplies soit par l'eau potable, soit l'eau sanitaire, d'irrigation, etc... Analyse de fonctions qui aboutira à une valorisation spécifique prenant en compte le cycle complet de l'eau. Ce n'est que lorsqu'on aura fait cette demande que l'on pourra parler du produit eau à part entière.

Nous avons prononcé deux mots : valeur et fonction, par eux ne peut-on pas communiquer de façon très large, car l'eau concerne tout le monde.

Peu de produits peuvent se prévaloir d'un usage obligatoire, donc utilisons le pour repositionner l'eau dans notre vie, dans la campagne, dans la cité. Voilà ce que je voulais vous dire en introduction, parce que je pense que, au travers de la méthodologie que je vais vous présenter, l'eau peut en faire l'objet d'une excellente application.

Je ne vais pas être trop long avec la méthode, il faut quand même que vous en connaissiez les éléments principaux. Quelques définitions d'abord.

\section{L'une est donnée par l'AFNOR :}

"L'analyse de la valeur est une méthode de compétitivité et de créativité visant à la satisfaction du besoin de l'utilisateur, par une démarche spécifique de conception à la fois fonctionnelle, économique et pluridisciplinaire".

L'autre est plus accessible au point de vie du langage :

"L'analyse de la valeur est une méthode pour concevóir, ou reconcevoir un produit, de manière qu'il assure, au moindre coût toutes les fonctions que le client désire e seulement celle-là, avec toutes les exigences requises et pas plus".

Sur la figure 1 ci-aprés, vous pouvez apercevoir, au travers de l'ère industrielle entre le début du siècle et 1980, les évolutions de la productivité au travers de l'usage de différentes méthodologies, qu ont permis de l'accroître. Ce qui est ambré sur le rectangle supérieur représente un décollage non négligeable et pouvant toujours s'améliorer, pourquoi ? Et bien, parce que la limite des méthodes O.S.T. pas exemple (organisation scientifique du tra. vail) est liée essentiellement à la perfor. mance de l'homme, dans les dispositifs industriels. Le contrôle de la productivite est lui aussi lié, en grande partie, à lé pertinence des gens qui sont sur les poste: de travail et qui réalisent, de façon plus or moins parfaite, un certain nombre de tâches.

L'analyse de la valeur se positionne différemment. Dans les définitions que vous avez vu tout à l'heure, on parle de façor continue de l'adéquation des bésoins de l'utilisateur et les fonctions remplies par le produit. A partir du moment où l'on adme que ce couple est variable, on peut faire évoluer la productivité au travers de celui. ci. Un exemple simplement pour dire que l'analyse de la valeur, est actuellement at Japon la plus florissante, chez Hitachi, elle représente à peu près $5 \%$ du chiffre d'affaire en amélioration annuelle.

Sur la figure 2 ci-aprés, vous voyer la place de l'analyse de la valeur dans tout le champ, depuis les clients qui sont le point de départ de toute action industrielle e commerciale, les besoins, les choix de production, la définition du produit ou la reconception progressive détaillée, le contrôle des productions, l'expérimentation.

Si nous regardons sur l'extrême droite de cette figure, vous voyez un rectangle mar ketting. Le marketting, on peut en donner la définition suivante : c'est une approche du produit au travers du marché, mais c'es aussi la possibilité de voir ce que demande. rons dans l'avenir les utilisateurs potentiels d'un produit, quelqu'il soit. Le marketting se situe en amont de l'analyse de la valeur. il donne des informations sur la prospective: vous voyez que, une fois les clients ciblés les besoins définis,ainsi que les choix de productions, définis en niveau, l'analyse de la valeur se positionne au niveau de la conception et de la reconception des produits.

Donc, lorsque la conception, ou la reconception du produit est effectuée, on parlera de contrôle des solutions que l'on a retenues, expérimentations, lancement du pro- 


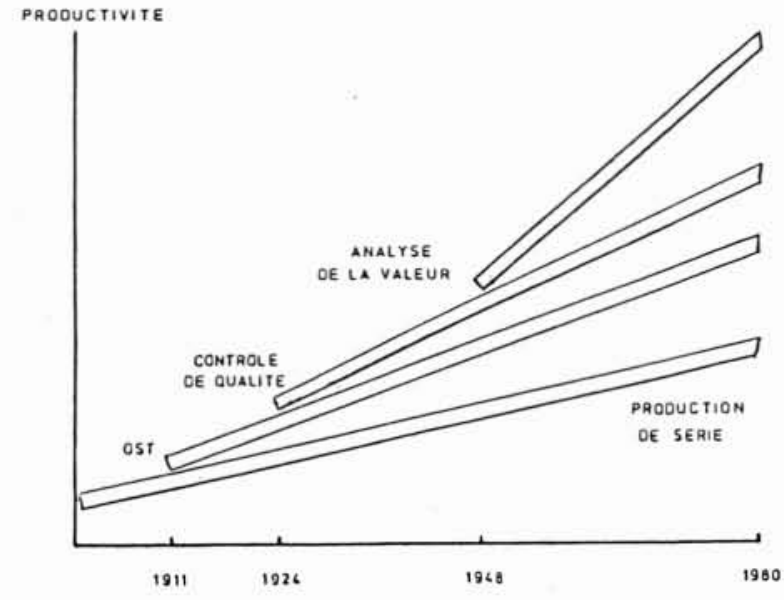

1. Résultats en fonction des différentes méthodes de rationalisation.

\begin{abstract}
CHAMP
LAAnalyse de la Valeut est essentiellement la e critique roconstructive , d'un systerme existant ou d'un projet dans ses stades successits d'elaboration - par controntation du systeme ou du projet a des fonctions el a des valeurs. Cependant, I'appellation - Analyse de ta Valeut, recouvie souvent toutes les techiniques daide à la conception economique : créativité, méthodes de recherches analvias. de classeron de doch menta matketing et methodes de gestion.
\end{abstract}

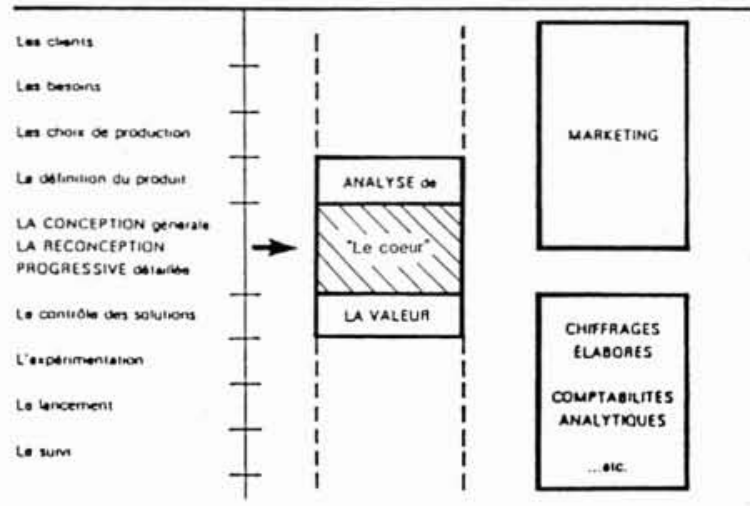

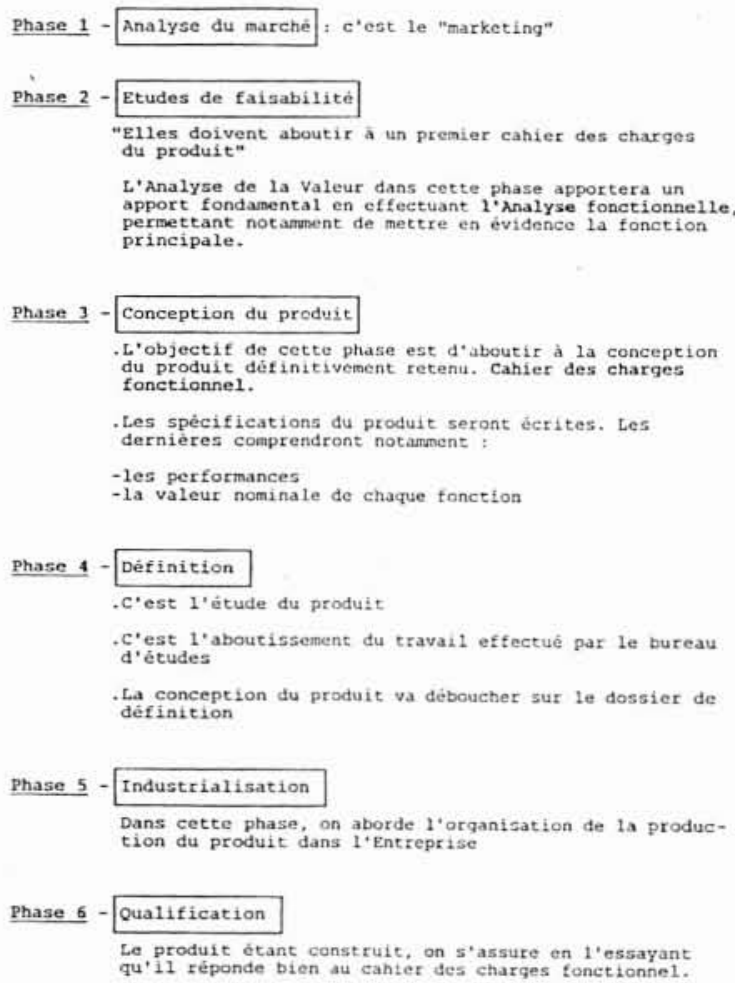

3. Les différentes phases de l'analyse de la valeur.

2. La place de l'analyse de la valeur.

duit et suivi de la production, et vous voyez que, au dessous du rectangle Marketting, dans un autre rectangle, on procède au chiffrage des différentes solutions. la comptabilité analytique permettant de vérifier les résultats.

L'analyse de la valeur, dans son déroulement (figure ci-aprés), se décompose en phases succéssives :

- La première phase est l'analyse du marché, elle va nécessiter des investigations qui peuvent être plus ou moins longues et plus ou moins coûteuses, selon les produits et le marché sur lequel vous vous situez. L'analyse du marché est indispensable pour demander une étude d'analyse de la valeur, quelle qu'elle soit. L'analyse du marché comprend également une analyse de tous les produits concurrents, assurant plus ou moins bien les fonctions du produit que vous possédez déjà ou que vous voulez réaliser.

- La phase 2, ce sont les études de faisabilité, c'est-à-dire les études permettant de mesurer s'il est industriellement possible de fabriquer un produit, de telle manière que l'on puisse apprécier si le prix auquel on pourra le mettre sur le marché est compétitif par rapport au prix que sont prêts à payer les clients pour ce produit.

- La phase 3 est la conception du produit, la conception au stade de l'ensemble de ses constituants. On va ici procéder de façon analytique à l'examen des différentes conceptions possibles.
- Phase 4 définition, c'est-à-dire que, une fois les concepts posés et entérinés par les personnes qui sont chargées de l'étude, on établira les cahiers des charges fonctionnels des différentes parties du produit.

- Les bureaux d'étude pourront travailler et nous passerons à la phase 5 , c'est-à-dire, l'industrialisation et la réalisation.

Si nous sommes sur des produits à haute technologie (par exemple, industrie aéronautique), on parlera de qualification, c'està-dire que, avant de mettre le produit sur le marché, on lui fera subir différentes séries de tests, pour voir s'il répond bien à toutes les exigences que le client attent de ce produit. Voilà ce que je voulais vous dire. 


\section{VIGNOLES}

\section{Nattero, nous vous remercions.}

Je pense que, autant l'exposé de $M$. Brigando que l'exposé de M. Nattero sur ces deux sujets sont très intéressants pour ce qui nous concerne, et je vais donc donner la parole à la salle pour les questions qui ne vont pas manquer.

\section{CHOCAT}

Je voudrais revenir sur le dernier exposé, sur l'analyse de la valeur, en complément, pour simplement signaler un point qui me parait extremement important, c'est les fonctions qui doivent être définies préalablement au processus de construction.

Je voulais faire remarquer que, dans les réseaux d'assainissement, la façon de définir les fonctions est tout à fait déterminante sur le type de solution que l'on peut être amené à proposer. Si on définit les fonctions $d u$ réseau d'assainissement comme étant des fonctions d'évacuation des eaux pluviales hors de la cité, ou des eaux usées hors de la cite, on aurra beaucoup de mal à avoir comme solutions des solutions d'infiltration ou de stockage local.

Je pense qu'en terme d'optimisation économique, ou de satifaction, comme je le définissais ce matin, le choix des differrentes fonctions que doit effectivement remplir, pour les différentes catégories d'usager, le systeme artificiel d'assainissement doit être mis au coeur du débat et l'on ne peut pas rester sur des définitions qui datent du $18 \mathrm{e}$ ou du 19 e siècle, qui étaient des définitions $d u$ courant hygiéniste qui consistaient d̀ dire, on va évacuer l'eau le plus vite possible, en mettant un tuyau et là, le problème était résolu. Je crois que les techniques d'analyse de la valeur vont permettre de redéfinir les fonctions mêmes que doivent remplir les systèmes d'assainissement.

\section{VIGNOLES}

Sije comprends bien tu fais apparaitre.le fait que l'évacuation seule des eaux pluviales vers le milieu naturel ne constitue pas l'optimum économique, et qu'il faut à chaque fois réenvisager d'autres solutions (rétention, etc...) qui permettent de retenir les flots et de minimiser les coûts d'investissement, par exemple.

\section{CHOCAT}

Je ne sais pas si ce sont les seules solutions, simplement par analogie, si l'on avait posé un jour la question d̀ une entreprise "inventez-moi un savon qui ne fonde pas sous la douche", personne

\section{Discussion sur les exposés de Messieurs M. BRIGANDO et R. NATTERO}

n'aurait inventé le savon liquide. Je crois que la question n'était pas d'inventer un savon qui ne fonde pas sous la douche, la question était d'inventer un savon que l'on puisse utiliser sous la douche et je crois qu'en matière d'assainissement, c'est pareil. Si l'on essaie d'optimiser un réseau d'évacuation des eaux, on ne regarde peut étre pas au vrai problème.

\section{VIGNOLES}

En gros, on cherche à avoir un réseau qui permette de répondre à la fonction de non inondation de zone, et de non pollution, mais qui réponde aussi avec un critère économique qui soit des plus satisfaisant.

\section{CHOCAT}

Le critère économique fait partie des fonctions, de toutes façons.

\section{VIGNOLES}

Ceci étant précisé, quelles sont les autres questions?

\section{Mme COGEZ}

Je ne sais pas s'il s'agit d'une question, mais plutot d'une remarque. On est resté silencieux, après cet exposé sur I'A.V., parce que ce n'est pas du tout le langage qu'on emploie tous les jours, et on voit, avec ces deux exposés qui abordent tous les deux la même idée, c'est-à-dire, l'approche économique par rapport à un aménagement pour les eaux pluviales, on a deux approches que l'on a du mal à relier, car il est vrai que le coût des ouvrages dépends de tous les paramètres dont nous a parlé $M$. Brigando; il est vrai aussi qu'on peut imaginer de réfléchir à un aménagement avec les méthodes d'A. V., mais, aujourd'hui, nous techniciens, on ne sait pas faire, on va repartir en réfléchissant beaucoup, je ne sais pas si d'autres, dans la salle, ont la même impression.

\section{TORTEROTOT}

Finalement, ce qui ressort et B. CHOCAT émet la même observation dans sa remarque, c'est qu'on aurrait pu aborder le problème de la même manière dès la première présentation de ce matin; on a l'impression de la part de quelqu'un qui ne maitrise pas l'hydrologie urbaine, comme c'est mon cas, qu'on n'est pas tout à fait sûr d'être fixé sur les objectifs que l'on veut atteindre, c'est-à-dire quà la fois, on cherche des criteres pour füxer les meilleurs objectifs, mais on a également besoin de trouver les objectifs pour connantre les critères et comment les employer.

Où est l'intérét collectif? On n'a pas parler du problème d'équité. L'objectif est-il de diminuer les inondations ou de répartir uniformément le risque, en sachant qu'on l'amoindrit, à priori, sil'on se débrouille pas trop mal.

Or, pour toute démarche économique, il faut que l'on füxe, dès le départ, un certain nombre de principes et d'objectifs et qu'après, on applique les arguments.

Alors, on peut poser la question à ceux qui font de l'hydrologie urbaine: quels sont les objectifs et les critères pertinents ?

\section{VIGNOLES}

Je pense qu'effectivement, c'est une bonne question. Il y a sans doute dans la salle bon nombre de gens qui ont cette conscience : on n'est pas encore dans I'histoire de l'assainissement, on sort de la préhistoire et des collectivités viennent de s'apercevoir qu'il existait un certain nombre de ruisseaux souterrains que l'on appelle les égouts, et que dans ces égouts là, l'eau circule, que cette eau peut etre intérressante, qu'il faut employer des gens pour les exploiter, c'est cela la réalité d'aujourd'hui.

Alors, si vous supposez quà votre place, il y a un parterre d'élus, à propos d'A. $V$., ces gens se demanderaient sils sont en train de faire un cauchemard, ou sils font un beau rêve. Je crois que c'est un décalage par rapport au terrain mais comme le soulignait $C$. Cogez, ce qui est important, c'est que, entre les deux exposés qui viennent d'être entendus, on parle de la même chose, il y a d'un coté une approche dont on $a$, en tant que technicien, l'habitude, d'un autre coté, il y a une approche nouvelle, qui me parrât particulièrement intéressante, parce qu'elle ouvre aussi des perspectives et qu'elle est peut-être la façon de faire passer un certain nombre de techniques dites nouvelles ou alternatives qui sur le terrain se développent relativement mal.

Il y a quelques essais mais on ne peut pas dire que la vaccination des techniciens 
ait été opérée. Alors, I'A.V. est peut-être aussi le moyen d'y arriver.

\section{DASSONVILLE}

Ce matin, je suis intervenu en temps que technicien; alors cet après-midi, puisque vous parlez des Elus, je met ma casquette $d$ 'Elu, pour vous interroger à propos de I'A.V. Aujourd'hui, c'est vrai que beaucoup d'Elus ont l'attention attirée par les architectes, par les urbanistes; à propos des techniques d'A.V. Je m'y suis un peu isntéressé à ce jour; mais à vous écouter, je m'interroge sur la raison qui vous a amene à déclarer tout à l'heure que le domaine de l'eau est typiquement un domaine où I'A. $V$. doit s'appliquer.

Alors, jaimerais vous entendre là-dessus nous apporter quelques précisions.

\section{NATTERO}

Ce qui me fait dire cela, c'est que l'eau, comme je l'ai dit au début, est un produit indispensable à la vie. Mais, à contrario, pour nous tous ici, parce que nous avons de l'eau partout dans la rue, il en coule pour laver les trottoirs, on en a au robinet, à la cuisine, à la salle de bain, etc..., on ne la considère plus tout à fait, de mon point de vue, comme un véritable produit d̀ part entière et je crois que, si nous voulons donner à l'eau la place qu'elle mérite, il faut la repositionner par rapport aux fonctions qu'elle remplit, aux fonctions vitales qu'elle remplit, pour la vie humaine.

Vous n'avez quà aller dans des pays où l'eau manque, où l'eau est polluée, il y a de grands pays d'Europe où l'eau est complètement polluée, où on ne peut pas boire l'eau que l'on voit courir dans les ruisseaux; on a alors une impression complètement différente et les rapports entre l'homme et l'eau changent complètement.

\section{DASSONVILLE}

Pardonnez-moi, je ne suis pas sûr d'avoir compris, et comme le disait à l'instant le Président de séance, jaj l'impression de vive un cauchemar. $A$ vous entendre, lorsque vous dites "on", de qui s'agit-il ? S'agissant du produit eau, on a bien en face de nous des consommateurs, donc des gens qui acceptent de payer un certain prix pour le produit qui leur est livré, disons quá la notion de produit, aujourd'hui, celle de service est venue prendre place. Alors de qui s'agit-il, lorsque vous dites "on"?

\section{NATTERO}

J'ai voulu parler des differentes formes d'eau, j’ai parlé d'eau potable, j'ai parlé d'eau industrielle, d'irrigation, d'eaux sanitaires, on ne peut pas donner le même poids aux fonctions que remplissent ces différentes formes d'eau. L'eau à laquelle vous faites allusion, je pense, c'est l'eau qui coule au robinet et qui est potable en France. C'est la seule qui est payée vraiment par le particulier, cette eau à laquelle vous faites allusion.. Les autres formes de l'eau, je ne dis pas que les industriels ne payent pas certaines redevances en fonction de l'eau qu'ils utilisent, mais je ne suis pas certain qu'il $y$ ait un rapport judicieux entre les prix et les fonctions remplies industriellement par l'eau qu'ils utilisent. C'est un exemple.

\section{AMORY}

Jaurais effectivement une question à poser sur I'A.V. que jai essayé d'utiliser dans l'eau pluviale.

II y a un petit probleme pour chiffier, car personne ici ne peut chiffrer le surdimensionnement des réseaux. En effet, I'A. V., ne peut s'appliquer que lorsqu'on a une maftrise parfaite de la technique. On pourrait parfaitement l'appliquer ponctuellement et pas globalement comme vous l'avez dit, dans le domaine de l'eau, en effet comment peut-on avec I'A. $V$., introduire une technique de l'aléatoire, car quelle est la définition du besoin dans le domaine des eaux pluviales, si on demande d̀ un Elu, sa problématique, c'est quill n'y ait jamais d'inondations. Effectivement, sur le plan technique, on sait très bien que tout phénomène qui peut avoir lieu, aura lieu un jour, c'est ce "un jour" que l'on ne connân jamais.

Quand on parle d'une pluie décennale, on sait très bien que ce n'est pas tout les dix ans qu'elle se reproduit, évidemment, quand on dit qu'elle est centenaire, elle peut se reproduire 10 fois de suite. Donc effectivement, jai très peur de I'A.V. et je préfererais répondre comme les urbanistes qui disent : lorsque je fais un plan d'urbanisme, je ferais mieux de laisser un espace réservé, dans lequel je ne sais pas exactement ce que je vais faire, car lavenir parlera.

Je crois que c'est une prudence d̀ laquelle on ne doit pas échapper. Alors, je m'inquiète. Je dois dire que Brigando, par exemple, fait de I'A.V. sans le vouloir, seulement dans l'exposé de Brigando, on reconnâft bien là l'expérience des choses qui nous ont fait mâtriser petit à petit, une technique que l'on ne maîtrisait pas.

Je ne sais pas si c'est un paradoxe, c'est le même paradoxe qui consiste à dire que ce n'est pas parce que la vie n'a pas de prix qu'elle n'a pas de valeur. Maintenant, si l'on reprend l'A.V., si j’ai bien écouté la défini- tion, on peut très bien lappliquer dans les réseaux, dans l'exploitation des réseaux, dans la sécurité, dans une station d'épuration, mais alors, je serais terrorisé si on l'employait sur une idée globale de la conception de l'évacuation de l'eau. Je pense que l'on retomberait dans la même erreur dont vient de parler M. Chocat, je crois, c'est-à-dire que lorsque l'on veut trop serrer l'enjeu, on serre trop la machine et elle ne marche plus.

Jai envie de dire que, dans laléatoire auquel nous avons à faire face et dans l'évacuation de l'eau, il faut être très prudent, que ce n'est pas l'A.V., bien qu'il faille des méthodes assez larges, et j'allais dire le plus large possible, qui peut répondre à nos besoins, car nul ici ne peut chiffrer un surdimensionnement, pour l'excellente raison qu'il n'y a pas d'inondation, mais on peut chiffrer un sous-dimensionnement.

\section{NATTERO}

Je voudrais répondre parce que là, vous posez un probleme de fond, en ciblant un petit peu I'A. $V$. comme étant applicable à certains problemes, relativement bien circonscrits et pas à d'autres.

Je ne partage pas du tout votre point de vue, je vous prie de m'en excuser, mais je crois aussi que vous etes excusable, parce que la présentation de I'A.V. que jai faite n'est pas une présentation faite pour vous apprendre à faire de I'A.V., mais simplement vous sensibiliser à cette approche et je n'ai pas eu la prétention en cinq minutes de vous donner toutes les composantes qui se cachent derrière cette méthodologie.

Dans la salle, ily a une personne, je crois que je peux la nommer M. CALVET, avec qui jai eu l'occasion de faire de I'A. $V$. et de pratiquer vraiment cette méthodologie. Je crois que c'est une méthode, contrairement à ce que vous avez dit, qui permet précisément de chiffrer l'inchiffrable, dans un certain nombre de limites, bien entendu.

Vous parlez des crues décennales pour certaines régions par exemple.

Rien n'empeche de chiffrer ce que jappellerais une fonction sécurité que vous souhaitez donner au dimensionnement des réseaux que vous allez mettre en place, je ne connais pas votre métier, donc je ne me hasarde pas trop dans le domaine des termes techniques que vous utilisez, mais il est parfaitement possible de mettre en évidence cette fonction sécurité.

Je vais prendre un exemple limite qui illustre l'intérét de I'A.V. Savez-vous qu'Ariane, si on la tire, c'est grace à I'A.V.? 


\section{VIGNOLES}

Je crois qu'il serait peut-être important, en complément, que lon entende $\mathrm{M}$. Calvet, pour qu'il contribue à faire le fil conducteur entre la "valeur" des mécréants que nous sommes et l'A. $V$.

\section{CALVET}

Tout à fait, l'expérience à laquelle fait référence $M$. Nattero est une expérience qui consiste à faire émerger dans le tissus de la Société du Canal de Provence, au milieu des différents services, un produit qui serait un produit gestion automatisée appliquée aux réseaux d'irrigation; c'est à la fois très simple et très compliqué.

\section{NATTERO}

M. Calvet, vous pourriez peut-être, sans dévoiler le contenu précis de ce que nous avons fait, dire au moins combien au cours de la première journée, les personnes qui étaient autour de la table étaient réticentes à cette approche, parce que justement, elles reprenaient des propos à peu près analogues à ceux qui ont été tenus tout à l'heure avec les problèmes de dimensionnement.

\section{CALVET}

Il a fallu, en effet, trois séances, espacées d'un mois, pour qu'effectivement un certain nombre de réticences se dissolvent et qu'apparaisse l'intérêt de rentrer vraiment dans la démarche.

\section{VIGNOLES}

Si je comprends bien, la salle offre un phénomène de répulsion des plus classiques quand vous abordez le sujet.

\section{NATTERO}

Je suis habitué à ce genre de choses, c'est tout à fait normal, parce que je crois que cela bouscule notre propre mode de fonctionnement, on n'est pas habitué à raisonner de façon dématérialisée, on pense tout de suite à la solution et au travers des questions qui m'ont été posées, transparaissaient, de toute part, les réponses auxquelles les personnes pensajent et c'est tout à fait humain, je l'ai vécu moi-méme lorsque je ne faisais pas encore de I'A.V. Ce que je peux vous dire encore, c'est que je pratique I'A. V. depuis un quinzaine d'années et c'ést la seule méthode organisationnelle avec laquelle je n'ai jamais eu d'échec.

\section{AMORY}

Il ne faut pas qu'on ne la pratique pas, il ne faut pas dire que l'on en a peur, c'est justement dans la gestion de lincertitude que l'on n'est pas encore tout à fait satisfait, parce qu'en fait, lorsque vous faites un projet, on va parler d'Haussmann, par exemple, les répercussions vont se faire pendant combien de temps? Alors je peux très bien faire de l'analyse de la valeur, je serai sanctionné peut-être dans cinquante ans parce que jaurai eu la chance de ne pas avoir de pluie décénale pendant ces cinquante ans.

Il ne faut pas nous faire dire ce que nous ne disons pas. Déjà, nous sommes très méfiants sur la technique que l'on va apporter et que justement I'A.V. va nous aider à mieux maftriser. Parce que tout à l'heure, M. Calvet a parlé de quelque chose de ponctuel, on n'a pas dit globalement la gestion de l'eau. La gestion de l'eau, faite globalement par I'A.V., je ne suis pas encore tout à fait convaincu. Par contre, dans la technique que l'on a à employer pour évacuer l'eau, peut-être on évacuerait l'eau beaucoup mieux parce qu'on sait qu'il y a d'autres raisons, d'autres partenaires, il y a des tas de choses que l'on peut encore découvrir, et ģa c'est vrai que I'A.V. peut nous le donner.

\section{NATTERO}

Si vous permettez, je vous reprends. Vous cantonnez l'application possible de I'A.V. à une certaine forme d'eau. Lorsque je parle de l'eau en tant que produit global, c'est parce que, à l'origine, l'eau, à quelques réserves près, est une eau qui peut être appliquée à différents besoins, je dirais même à tous les besoins avec quelques restrictions s'il s'agit d'une eau plus ou moins chargée en ceci ou cela, mais au stade naturel.

Mais pourquoi ne voulez-vous pas appliquer cette approche différemment à l'eau potable, à l'eau d'irrigation, aux eaux industrielles, je ne vois pas pourquoi il faudrait que l'on exclut l'application de cette méthodologie aux eaux usées par exemple, et je crois que chaque mode d'utilisation de l'eau nécessite une approche différentielle du problème de l'eau par la méthode de I'A. $V$.

\section{CHOCAT}

Ce que I'A.V. peut permettre, c'est de trouver une bonne solution, sans prétendre le moins du monde que cette solution est la meilleure.

\section{NATTERO}

La solution que l'on adoptera est celle que la collectivité, ou l'indistriel, ou le particulier est prêt à payer et uniquement celle-là.

\section{CHOCAT}

La définition de la qualité, il faut peutetre la rappeler, c'est l'aptitude d'un objet à répondre $\grave{a}$ un momment donné d̀ un ensemble de fonctions choisies par les utilisateurs et ceci pendant une période donnée. Je crois qu'il y a une multiplicité des fonctions, une multiplicité des utilisateurs et puis le temps qui apparaît de façon différente à l'instant où I'on choisit la solution et il faut savoir avec un peu de modestie, que la solution ne sera vraie que sur une certaine période.

\section{BARRAQUE}

Par rapport à cette discussion, voici le point de vue du sociologue, si ce n'est pas complètement interdit dans cette salle. Je voudrais faire deux observations, plutot que de poser une question.

La première, je crois que ce qui me paraît le plus intéressant, le plus important dans la démarche qui nous est proposée, ce n'est pas la quantification, fa c'est un résultat à mon avis secondaire (je dis bien que jai le point de vue du sociologue en disant cela). Ce qui est important, c'est d'arriver à réunir autour d'une table différentes personnes qui sont partie prenante dans le sujet dont on discute, et d'arriver à obtenir qu'ils discutent objectivement. Et pour ce faire, il faut obtenir des participants qu'ils se mettent à distance de l'objet, qu peut-être l'objet de leur travail. Et je crois que c'est là qu'est le problème. Si on y arrive, c'est très bien, mais ce n'est pas obligatoire que l'on puisse y arriver.

Je pense que l'un des aspects essentiels du travail des gens qui font de I'A. V., est partagé par tous les gens qui font du conseil en organisation, de la formation institutionnelle, avec des techniques très différentes. Le problème, c'est d'arriver à obtenir que les gens puissent prendre un minimum de distance par rapport à ce qu'ils font, pour pouvoir réfléchir collectivement. Cela fait partie, je crois, de la demande fondamentale d'une société qui se voudrait rationnelle et moderne, mais je pense que la réticence qui s'exprime dans la salle, peut etre double. Elle peut être une ressistance d'ingénieur et de technicien.

Si on avait ici des ouvriers d'assainissement, il serait très difficile d'obtenir d'eux qu'ils se distancient de leur travail, parce qu'ils ont tendance à valoriser le fait qu'ils font un métier dangereux, difficile.

Si vous me donnez une petite seconde, je vais vous donner un exemple qui n'a rien à voir, dans un tout autre domaine, car quand vous descendez dans une mine de charbon, vous rencontrez des ouvriers qui savent très bien qu'ils vont mourir de la silicose, 
beaucoup plus vite que d'autres gens. Mais la silicose est un sujet tabou. Par contre, ils savent très bien mobiliser la silicose, quand ils vont en négociation avec leur patron, en tant que syndicat, mais pas forcément pour obtenir quelque chose dans le domaine de la silicose, autrement dit, quel est le sujet de la négociation que l'on fait ? Est-ce que lobjet est vraiment lobjet direct, précis sur lequel on discute, fa, c'est la demande de la rationalité, ou n'y a-t-il pas des arrières pensées?

Tout le problème de cette demande est de faire en sorte que les gens n'aient pas d'arrière-pensées et qu'en plus, ils se différencient de ce qu'ils font. Et f̧a, ce n'est pas évident et c'est là qu'est la deuxième difficulté. Quand on a affaire, et je pense que c'est là la réticence qui s'exprime dans cette salle, à l'eau, vous parlez de l'eau répondant à des besoins, donc dans une optique fonctionnelle.

Ce sont des besoins d'eau domestique, c'est des besoins d'irrigation, c'est des besoins d'eau industrielle, c'est des besoins de lutte contre la pollution. Mais, l'eau, c'est tout, c'est la vie, c'est un des éléments symboliques qui rendent effectivement lappréhension fonctionnelle probablement plus difficile que pour d'autres produits, comme par exemple la fusée Ariane. Mais, je ne veux pas particulièrement trancher à l'encontre de ce que vous faites, je pense que c'est particulièrement difficile.

\section{NATTERO}

Je crois que vous avez enrichi d'une certaine façon ce que je n'ai peut-être pas suffisamment dit, mais, lorsque vous dites "'eau c'est la vie", c'est ce que jai dit et c'est bien pour cela que je déclare que l'eau, il faut pas la prendre en tant que produit global, et qu'il ne faut se contenter de traiter l'eau une fois parce que c'est de l'eau d'assainissement, une autre fois parce que c'est de l'eau d'irrigation, etc...

Et, lorsque vous dites que les groupes d'A. $V$. permettent aux gens de communiquer entre eux, même sur des sujets qui ne les intéressent pas forcément au quotidien, cela a une importance dans l'entreprise car cela créé des liens entre individus qui parfois ne s'étaient jamais parlé.

\section{BARRAQUE}

Cela peut parfois déboucher sur une cristallisation de conflits, ce qui parfois n'est pas une mauvaise chose.

\section{NATTERO}

Ecoutez, jai en permanence des groupes d'A. V., jai peut-être eu de la chance, mais je n'ai jamais débouché sur des contlits.

\section{VIGNOLES}

Je crois que cet exposé sur I'A. V. aura eu le mérite de nous la mettre dans l'esprit, et jespère que tous les participants dans cette salle, quand ils auront un probleme $\dot{d}$ traiter, auront le déclic $A$. $V$.

Où le déclic les mènera? II faudrait une séance supplémentaire pour avoir la réponse; cette analyse de la valeur $m$ 'a personnellement mis l'eau à la bouche.

D'autres questions?

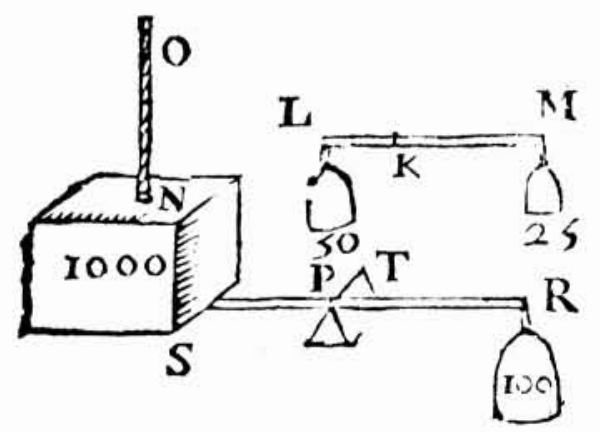

\section{NATTERO}

Suite à ce que vous venez de dire, il est bien évident que pour faire une sensibilisation à la méthodologie, il faut au minimum une matinée, ce qui permettrait d'avoir des échanges riches avec les participants et que je puisse exposer dans les détails, chacune des phases de la méthodologie et je me sers pour cela d'un exemple concret, soit que la salle m'apporte, soit que japporte au travers de cas que jai pu traiter.

Je voudrais dire aussi, et c'est la dernière chose que je dirai, c'est que, pour faire de I'A. V., on n'est pas obligé d'être un spécialiste du métier de l'eau, de l'électronique, de l'aérospatiale, ce n'est absolument pas nécessaire puisque justement, dans la démarche, on commence par dématérialiser le produit.

\section{M.VIGNOLES}

Merci MM. Nattero et Brigando pour ces deux exposés, je vais demander à $M$. Thibaud de me rejoindre à la tribune.

Je vais enchainer directement sur un exposé que je vais vous présenter, sur le bilan d'exploitation, et son utilisation.

Bon, je crois que l'on va revenir à des choses plus directes, plus concrètes; le bilan d'exploitation, cela va donc être une présentation concernant Toulouse.

Ce n'est pas non plus, un fait historique; je vous ai expliqué tout à l'heure que nous passions d'une régie directe à une concession à la C.G.E., mais j'espère que la qualité de l'exploitation n'en souffrira pas. 

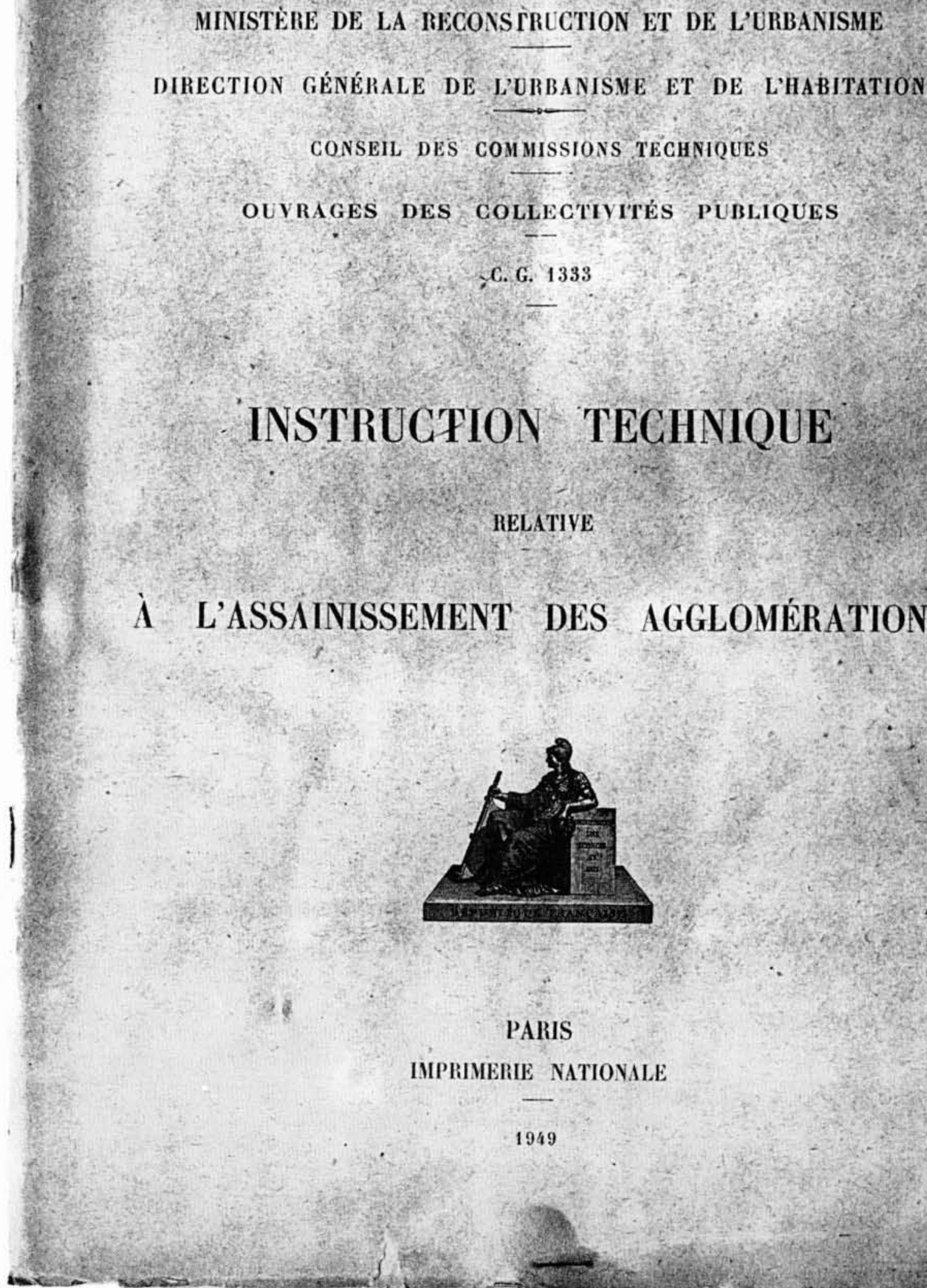

\section{3h 2 RELATIVE \\ A L'ASSAINISSEMENT DES AGGLOMÉRATIONS}

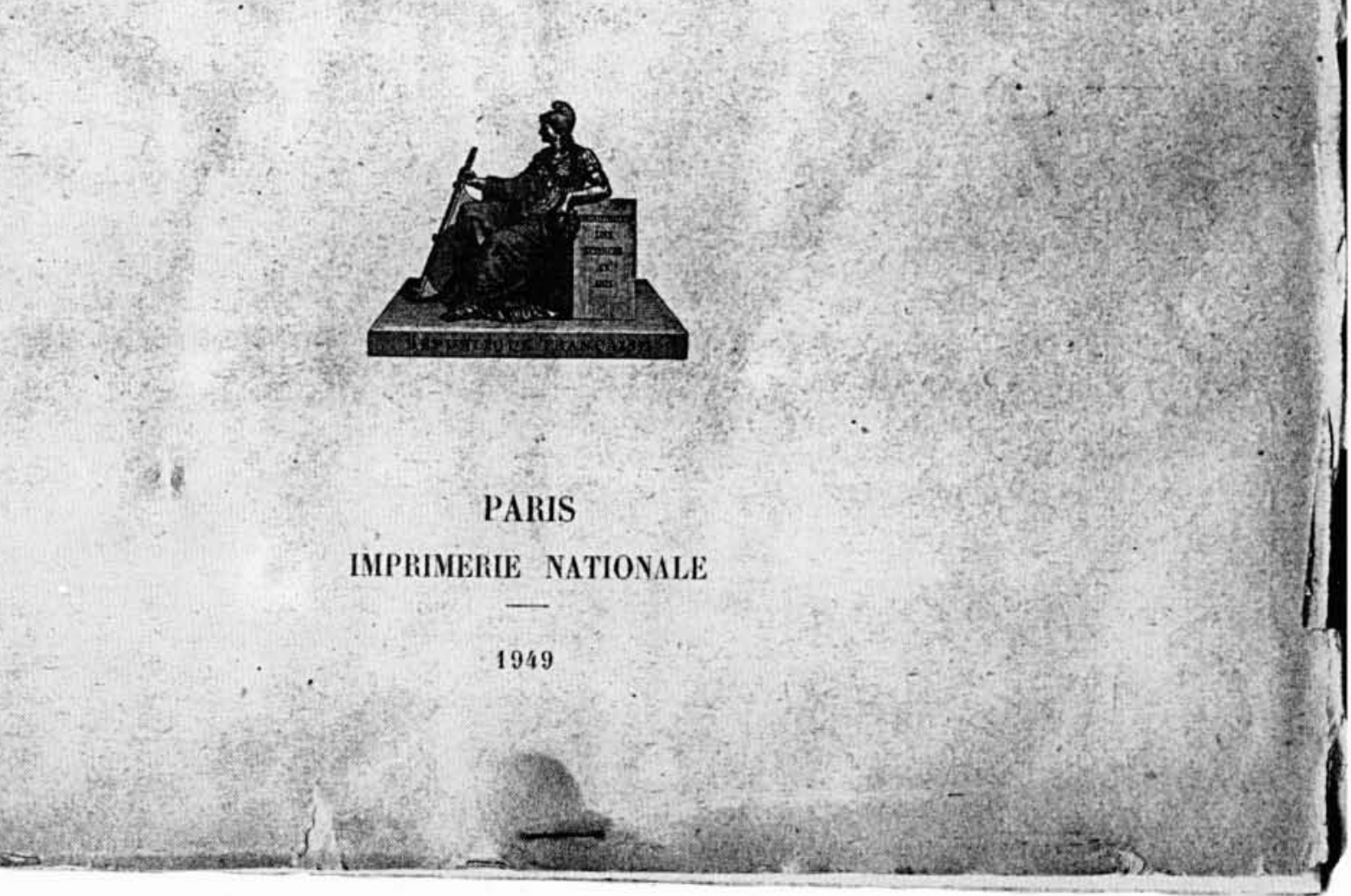




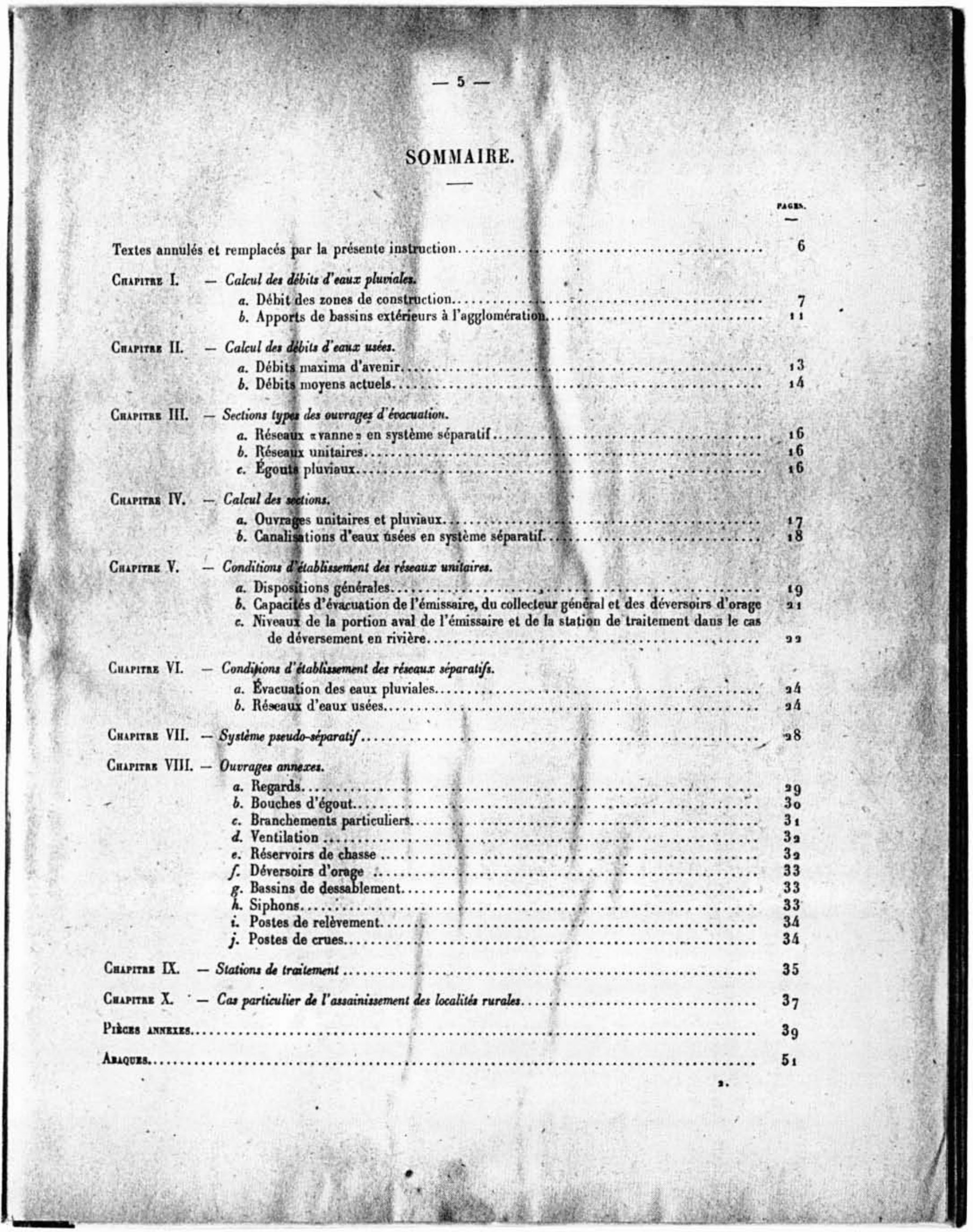




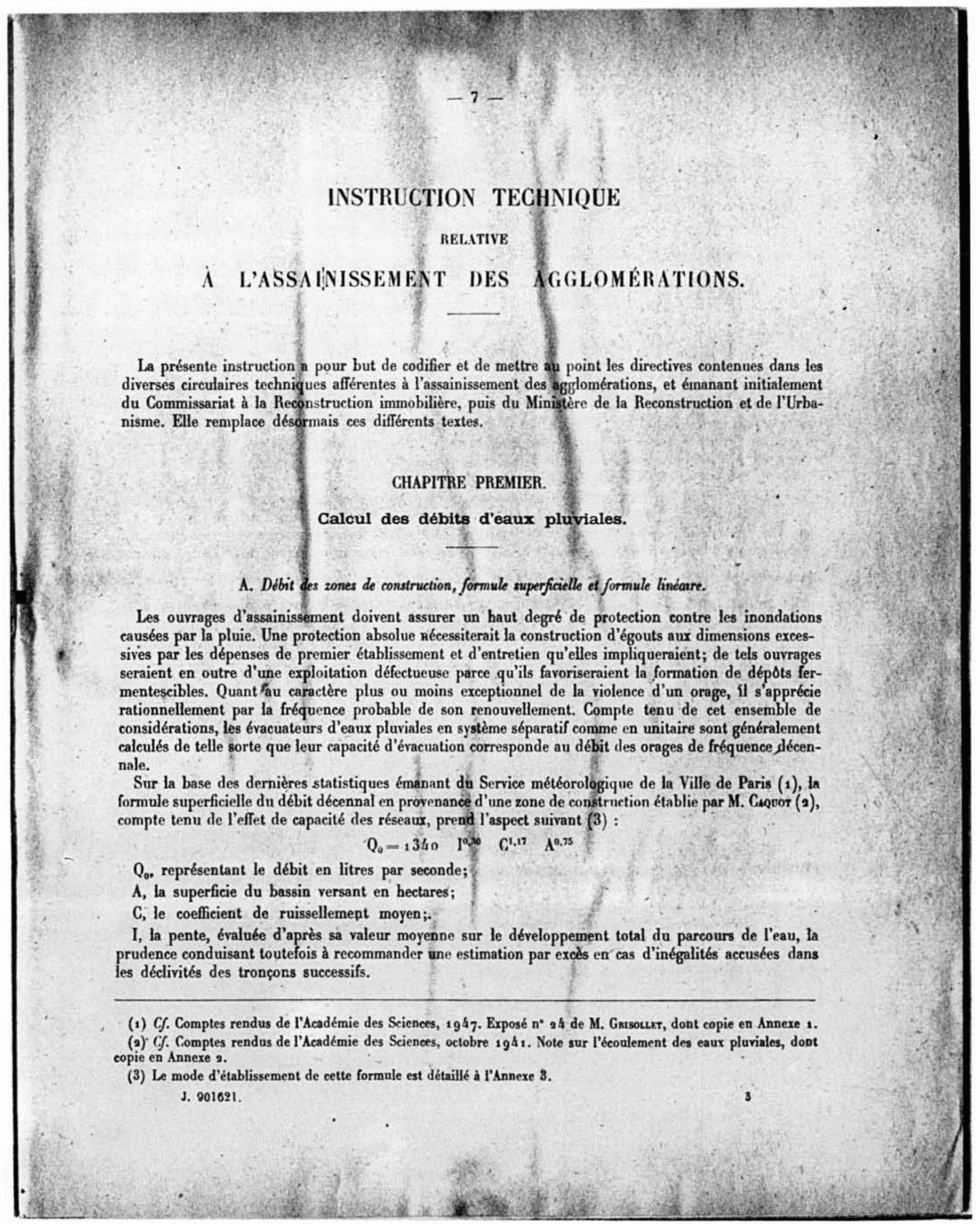

